\title{
II mantra degli ossimori colpisce la sanità
}

\author{
di Elio Borgonovi
}

Da alcuni anni la letteratura manageriale, ma non solo quella, sembra essere caratterizzata da un mantra, una specie di formula magica ripetuta costantemente: la soluzione di molti problemi della società va ricercata nella capacità di coniugare o rendere compatibili gli opposti. Un mantra che viene coniugato per diversi problemi critici della società e dell'economia, molti dei quali riguardano anche i sistemi di tutela della salute.

Una prima declinazione fa riferimento al rischio di fallimento dei sistemi economici, in particolare alla non sostenibilità dei sistemi di tutela della salute universalistici. Il mantra consiste nel sottolineare che si tratta semplicemente di una percezione in quanto il rischio di non sostenibilità deriverebbe non tanto da condizioni oggettive, quanto dalla divaricazione tra il rapido aumento dei bisogni e delle aspettative dei pazienti e un più lento adeguamento delle risorse. Il mantra consiste nel proporre soluzioni capaci di contenere le aspettative e agire contestualmente sull'aumento delle risorse e, dove ciò non sia possibile, nel forte recupero di efficienza. Infatti un certo numero di analisti e studiosi sostiene che le prime risorse "addizionali" sono quelle che non si sprecano. Tuttavia a nessuno può sfuggire che ricercare la compatibilità è "più facile a dirsi che a farsi", anche perché aumentano contestualmente le esigenze di altri settori di intervento pubblico quali istruzione, ricerca, mobilità, tutela dell'ambiente, cultura ecc.

Una seconda declinazione, che è ormai un classico della letteratura manageriale, nasce dalla interpretazione della parola rischio che in lingua cinese ha il doppio significato di minaccia e opportunità. Il buon manager è chi riesce a far prevalere la componente di opportunità, di innovazione e di spinta al cambiamento rispetto a quella di minaccia che è causa di arroccamento su situazioni consolidate, su modelli assistenziali sempre più inadeguati. Ma affinché prevalga la componente positiva è necessario agire sulle persone, sulla loro capacità di accettare la sfida del cambiamento. Cosa non facile quando entrano in gioco centinaia di migliaia di medici, di infermieri, altri professionisti della salute, altro personale amministrativo e tecnico e centinaia e migliaia di centri decisionali che interagiscono in senso gerarchico (Stato, Regioni, aziende sanitarie o aziende ospedaliere, unità organizzative all'interno delle aziende) $o$ orizzontale (rapporti tra le Regioni, rapporti tra aziende territoriali e aziende ospedaliere della stessa regione, rapporti tra aziende sanitarie/enti locali/organizzazioni che operano nel privato sociale).

Per favorire l'orientamento verso le opportunità viene enunciata la terza declinazione del mantra che si è diffusa nella letteratura manageriale soprattutto nell'ultimo decennio, quella del cosiddetto ambidestrismo, cioè capacità di coniugare l'orientamento all'innovazione e alla creatività (exploration) con quello della stabilizzazione e del consolidamento (exploitation). È un mantra che viene coniugato sia con riferimento a attitudini, atteggiamenti, comportamenti degli individui sia al funzionamento delle organizzazioni che devono essere strutturate in modo da favorire/incentivare/riconoscere persone o gruppi responsabili dell'una o dell'altra funzione. Per le patologie acute la risposta a questa duplice esigenza può essere individuata nella concentrazione in pochi grandi ospedali/aziende sanitarie della funzione di ricerca e dell' innovazione dei modelli assistenziali, mentre a strutture di minore complessità può essere affidata la responsabilità dell'applicazione su vasta scala di modelli assistenziali consolidati. Appare invece più complessa la possibilità di rispondere contestualmente alla duplice esigenza nel caso dell'assistenza dei 
pazienti cronici, anziani, non autosufficienti. Per tali ambiti, la funzione di innovazione non può essere concentrata in poche realtà e, anche a causa di ritardi della ricerca, spesso la funzione di applicazione diffusa delle conoscenze non può essere standardizzata. Si può ricorrere al metodo delle "buone pratiche" (best practices), ma questi processi assistenziali si svolgono in contesti economico sociali e istituzionali assai diversi da quelli che caratterizzano l'assistenza per acuti negli ospedali e quindi la replicabilità delle buone pratiche è meno significativa rispetto ai protocolli diagnostici e terapeutici definiti per patologie acute.

La quarta declinazione, più tradizionale nella letteratura clinica e in quella manageriale, ma che oggi viene rilanciata in un contesto ben più complesso del passato, riguarda i principi di specializzazione e integrazione. Il progresso delle conoscenze spinge inevitabilmente verso una specializzazione sempre più accentuata, ma questa fa sorgere un altrettanto forte bisogno di integrazione. Tradizionalmente nel campo della clinica questa funzione è stata di volta in volta attribuita al medico di medicina generale, in ospedale e ai MMG e PLS nel territorio. Di fronte ai livelli di specializzazione molto più accentuati del passato, si ritiene che difficilmente un unico professionista possa svolgere adeguatamente la funzione di integrazione. Perciò sono proposti modelli organizzativi di approccio interdisciplinare al paziente sia per quanto riguarda il trattamento delle patologie acute, sia per quanto riguarda la presa in carico di pazienti cronici o multi cronici. Occorre peraltro sottolineare che il lavoro di équipe sempre più articolate e numerose, richiede non solo conoscenze, competenze, capacità specifiche tra loro complementari, ma anche, se non soprattutto, una grande capacità di "lavorare in gruppo". È facile sostenere che nell' approccio interdisciplinare il risultato è superiore alla somma degli apporti dei singoli professionisti, ma è altrettanto intuitiva la necessità di garantire comunque un riferimento unitario per il paziente, sia per quanto riguarda le tipiche funzioni mediche di diagnosi e cura, sia per quanto riguarda le funzioni di assistenza infermieristica. È pure facilmente comprensibile il fatto che l'integrazione risulti più facile in un contesto definito (per esempio il ricovero ospedaliero) rispetto a un contesto meno definito quale è l'assistenza sul territorio pre o post fase acuta per pazienti anziani, disabili, cronici, non autosufficienti.

Le considerazioni precedenti introducono la quinta declinazione del mantra, che consiste nel cercare di far convivere la standardizzazione dell'assistenza con la personalizzazione. La standardizzazione e la replicabilità di modelli assistenziali efficaci ed efficienti sono state e sono tutt'ora considerate uno degli strumenti più efficaci per ridurre $i$ costi unitari e ampliare il target di riferimento. Si pensi all'utilità di "fasce o limiti di normalità" dei parametri di salute (es. pressione arteriosa, colesterolo ecc.) utilizzati a fine di prevenzione, diagnosi e cura dei pazienti. Peraltro, va ricordato che un buon medico (e un buon infermiere per le proprie funzioni) non si limita a verificare che i parametri di un paziente rientrino nei limiti di normalità o presentino elementi di "non normalità" ma deve essere in grado di fare una valutazione specifica del singolo paziente sulla base della anamnesi o dei riscontri con precedenti situazioni. Inoltre, le nuove frontiere della medicina vanno nella direzione dei "trattamenti personalizzati" che sempre più saranno consentiti dagli sviluppi di nuove conoscenze quali terapie geniche e cellulari, medicina rigenerativa, nutraceutica. Di conseguenza la standardizzazione degli interventi di prevenzione, diagnosi, cura, riabilitazione potrà essere considerata solo come una soglia minima di accettabilità. Al contrario, la ricerca di più elevati livelli di appropriatezza ed efficacia dovrà essere affidata a un maggior grado e livello di differenziazione. Recuperare risorse economiche con i vantaggi della standardizzazione sarà condizione necessaria, ancorché non sufficiente, per conseguire obiettivi di sostenibilità nel breve e nel lungo periodo.

In tal modo si introduce anche la sesta declinazione del mantra, quella di far convivere le esigenze di breve e di medio/lungo periodo dei sistemi di tutela della salute. Soprattutto dopo la crisi degli anni 2007-2008 i vincoli del finanziamento pubblico, ma anche quelli di ampie fasce di popolazione colpiti da disoccupazione o riduzione dei redditi, hanno determinato interventi di contenimento della spesa. Ciò ha portato a privilegiare gli equilibri di breve periodo, penalizzando però le esigenze di medio/lungo periodo. Ridurre la prevenzione primaria, la diagnosi precoce, cure con costi limitati, sostituzione di attrezzature obsolete, uso di device e tecnologie a basso costo e di minore qualità a causa di vincoli della finanza pubblica o di vincoli dei bilanci familiari, può determinare un aumento di costi futuri ben più elevati. In particolare, i responsabili delle politiche sanitarie a livello nazionale, regionale e locale sono indotti ad adottare interventi che danno priorità agli effetti di breve periodo, mantenimento o recupero dell' equilibrio 
economico, poiché gli effetti di medio/lungo periodo non possono essere percepiti e sono comunque lontani nel tempo. Non di rado, essi ricordano a chi evidenzia questo rischio la arcinota frase di Keynes secondo il quale "nel lungo periodo siamo tutti morti" cui aggiungono quella secondo cui per arrivare nel lungo periodo occorre trovare equilibri nel breve periodo. Cosi impostato, il tema del breve e del medio/lungo periodo si pone in termini di trade off, ma il mantra spesso ripetuto consiste nell'affermare che dal modo in cui si affrontano le esigenze dell'equilibrio di breve periodo dipende la sostenibilità nel medio/lungo periodo. Migliorare la razionalità nelle politiche di contenimento della spesa consente di individuare condizioni più favorevoli per rendere compatibili la sostenibilità economica di breve e di medio/lungo periodo.

La settima declinazione del mantra riguarda la dimensione istituzionale del SSN, quella relativa al rapporto tra decentramento regionale e la corrispondente esigenza di coordinamento del livello centrale. Dopo un periodo nel quale ha prevalso la logica del decentramento regionale, oggi viene ribadita con sempre maggiore frequenza e forza l'esigenza di una centralizzazione delle funzioni da riportare nell' ambito delle responsabilità dello Stato per garantire l'uniformità dei cittadini e il coordinamento tra le regioni. È forte la critica sui 21 sistemi sanitari regionali rispetto a leggi fondate sui principi di universalità, equità, livelli essenziali di assistenza uniformi per tutti sul territorio nazionale. Il mantra consiste nell' affermazione secondo cui occorre ridare allo Stato maggiori poteri per contrastare situazioni di "differenziazione negativa tra le Regioni" (quelle dovute a inefficienza, sprechi e corruzione), senza penalizzare la "differenziazione positiva" (quella derivante da modelli di programmazione e di organizzazione dei servizi maggiormente conformi alle caratteristiche della popolazione e dei territori di riferimento). Per conseguire questo obiettivo non è sufficiente l'approvazione della riforma costituzionale e nemmeno di leggi di riforma del SSN, ma occorreranno decreti attuativi da parte dello Stato, leggi di applicazione da parte delle Regioni di una più elevata disponibilità sul piano politico e tecnico alla "negoziazione" tra i due livelli di governo nell' ambito della conferenza Stato-Regioni. Non basta il mantra per risolvere le questioni del "dialogo tra sordi" che spesso ha caratterizzato $i$ rapporti tra i due livelli di governo anche del recente passato.

L'ottava declinazione del mantra riguarda il rapporto tra tendenza verso le grandi dimensioni delle aziende sanitarie territoriali e delle aziende ospedaliere (o presidi) per conseguire riduzione di costi e contestuale sviluppo dell'assistenza territoriale che aumenta il numero e la varietà di punti di erogazione dei servizi. Al contrario di quanto avviene per altre tipologie di servizi (per esempio bancomat, erogatori automatici di bibite, accessi online a servizi standardizzati), nel caso della tutela della salute la maggiore capillarità del servizio non può far leva sulla automazione, salvo i casi di telemedicina. È difficile pensare che nel breve periodo si possa trovare un "giusto equilibrio" tra le due esigenze e quindi è forte il rischio che una delle due sia penalizzata, probabilmente quella della qualità dell'assistenza sul territorio. Per evitare la crescente divaricazione tra le dichiarazioni o la normativa e la concreta realtà occorrono "politiche attive" per lo sviluppo dei nuovi modelli assistenziali (continuità delle cure, assistenza territoriale, presa in carico dei pazienti cronici). Queste a loro volta richiedono investimenti non facili da attuare dati i vincoli della finanza pubblica.

La nona declinazione del mantra riguarda il rapporto tra eccellenza individuale ed eccellenza collettiva. Negli ultimi decenni si è progressivamente consolidata la ricerca delle eccellenze individuali a seguito dell'affermarsi di teorie sulla competizione come stimolo al miglioramento, sui champions da imitare, sui talenti o gli altri potenziali sui quali investire, sul riconoscimento del merito, sull'ascensore sociale, sul lavoro autonomo, sulla capacità di essere imprenditori di se stessi. Di fronte a evidenti distorsioni di questa cultura individualistica, molti hanno cercato il bilanciamento mettendo in evidenza che nelle organizzazioni complesse il miglioramento e il successo è dato dal gioco di squadra, dalla ricerca dell'eccellenza organizzativa o, più in generale, collettiva. Anche le analisi finalizzate a promuovere l'interdisciplinarietà e l'integrazione dei saperi (conoscenze e competenze) e delle capacità operative, hanno posto l'obiettivo dell'eccellenza organizzativa e collettiva. In particolare, nel campo della tutela della salute, il passaggio dalla centralità della malattia e dell'organo alla centralità della persona malata ha promosso l'introduzione di nuovi modelli assistenziali fondati sulla continuità delle cure, sulla presa in carico, sulla cooperazione tra vari professionisti. Tra l'altro, è interessante sottolineare che il termine cooperazione ha un significato più profondo della col- 
laborazione. Quest'ultimo richiama una attività congiunta di soggetti che intervengono e modificano la situazione di un oggetto (nel caso specifico il paziente come soggetto che diventa oggetto perché non interagisce), mentre il concetto di cooperazione richiama l'attività congiunta di soggetti che modificano non solo l'oggetto ma modificano anche le proprie conoscenze, competenze, abilità, comportamenti. Nella logica della eccellenza individuale e dell'eccellenza collettiva, il paziente interagisce con i professionisti che si danno carico della sua condizione di salute. Tuttavia non sembra risolta la contraddizione di istituzione di aziende pubbliche e private i cui vertici richiamano con frequenza "l'importanza del gioco di squadra", "la valorizzazione del contributo di ognuno", ma nelle quali gli incentivi sono collegati a performance individuali. Chi crede nella logica secondo cui è veramente eccellente chi è in grado di rendere eccellente il gruppo o l'azienda cui appartiene, dovrebbe essere creativo e innovativo anche nell'introduzione di sistemi capaci di considerare in modo equilibrato le performance individuali e le performance di squadra, di unità organizzativa, di azienda.

Una decima declinazione del mantra riguarda l'esigenza di rendere compatibili il principio di trasparenza e di diffusione dell' informazione per una migliore tutela della salute e quella di privacy. Oggi, informazioni sempre più specifiche e facilmente accessibili su malattie pregresse, trattamenti sanitari cui i soggetti sono stati sottoposti, parametri psicofisici, addirittura caratteristiche del genoma, sono utili per adeguare le risposte ai problemi di salute ai reali bisogni di ogni persona. Tuttavia, tali informazioni possono essere utilizzate anche "contro" le persone (per esempio discriminazioni nelle assunzioni, onerosità delle polizze per assicurazioni sanitarie ecc.) e pertanto si pone un grande problema di sicurezza dei dati. Forse esiste ancora una scarsa consapevolezza dei cittadini con riguardo alla tutela dei propri dati sanitari o riguardo alle informazioni che $i$ motori di ricerca possono trarre dai siti che vengono visitati. A sua volta, la normativa sulla privacy, soprattutto nel nostro Paese, spesso rende difficile attuare programmi di prevenzione mirati o addirittura ricerche finalizzate a meglio comprendere i caratteri epidemiologici della popolazione e la loro evoluzione. Per affrontare questo mantra occorre investire non solo nei sistemi informativi sanitari, che sono una precondizione per adottare modelli assistenziali più efficaci, efficienti, economicamente sostenibili, ma occorre investire anche nella sicurezza dei sistemi informativi rispetto ad attacchi esterni. Il tema diventerà sempre più rilevante con lo sviluppo di tecnologie (protesi, device, robotizzazione di alcune attività assistenziali, sistemi di intelligenze artificiali per la diagnosi e la decisioni di cure che possono subire interferenze di soggetti non guidati dal benessere del paziente) a funzionamento elettronico.

L'undicesima declinazione rappresenta un classico e riguarda il ruolo del pubblico e del privato. Secondo le teorie dominanti, l'intervento pubblico (nella forma di SSN, estensione a tutta la popolazione di fondi e casse, sostegno pubblico per la sottoscrizione di polizze assicurative obbligatorie come nel caso dell'Affordable Care Act degli USA) risponde ai principi di equità, solidarietà, tutela di un diritto umano, mentre l'intervento di strutture private nell'erogazione di servizi è motivato da un maggiore livello di efficienze. Si tratta di due impostazioni che hanno un non trascurabile contenuto ideale-ideologico. Infatti, vi sono ricerche empiriche che portano evidenze a favore o contrarie al livello di equità e solidarietà di sistemi pubblici caratterizzati da scarsa qualità delle politiche di tutela della salute e bassa efficienza delle strutture di erogazione dei servizi. Alcune ricerche hanno prodotto evidenze empiriche di una maggiore efficienza di erogatori privati rispetto a quelli pubblici, mentre altre non hanno riscontrato significativi differenziali di efficienza a parità di mix di pazienti o hanno rilevato differenziali di efficienza riconducibili esclusivamente o prevalentemente alla possibilità degli erogatori privati di selezionare la propria domanda. Pertanto negli ultimi anni vi è stato un riorientamento del dibattito poiché si evidenzia la contrapposizione tra soggetti pubblici e privati, che pur nel rispetto dei propri ruoli, sono caratterizzati da un funzionamento coerente con il fine di tutela della salute e soggetti pubblici e privati che invece perseguono obiettivi diversi, per esempio il consenso politico o la massimizzazione del profitto a danno della qualità. Questo riorientamento però non risolve il problema dell' individuazione di criteri e parametri largamente condivisi per distinguere i primi dai secondi.

La dodicesima declinazione del mantra riguarda la possibilità di rendere compatibili la tutela della salute e il consenso politico e, per quanto riguarda il privato, la ricerca di più elevati livelli di efficienza senza lo stimolo del profitto. Per quanto riguarda la prima parte del mantra, alcune teorie suggeriscono che un SSN in grado di garantire effet- 
tivamente i livelli essenziali di assistenza dichiarati definiti per legge previsti dai piani nazionali o regionali e una elevata percezione di funzionalità e equità contribuiscono ad aumentare il consenso degli amministratori. Tuttavia, tale relazione è molto indiretta ed è più facile che chi occupa posizioni di potere politico diretto e indiretto ricerchi il consenso tramite la concessione di piccoli favori o grandi benefici, per non parlare dei fenomeni di corruzione. La seconda parte del mantra sembra avere una maggiore consistenza in quanto nel settore di tutela della salute vi sono sempre state forme di volontariato e oggi stanno diffondendosi forme di imprese sociali in grado di garantire servizi di qualità a costi sostenibili anche da fasce economicamente deboli (low cost). Sono soluzioni che adottano modelli di business identificati come innovazione sociale o che rientrano nella vasta accezione di soluzioni di mercato immuni dalle distorsioni generate dalla massimizzazione del profitto.

L'elenco delle diverse declinazioni del mantra non è certo esaustivo ma è sufficiente per sottolineare due aspetti importanti. Il primo riguarda il fatto che le teorie e i modelli di analisi e di interpretazione che propongono di coniugare gli opposti, una volta elaborate, dimostrate e rese convincenti anche con ricerche empiriche, sono riprese e diffuse da molti ricercatori successivi non preoccupati di generare evidenze altrettanto solide e soprattutto diventano linguaggio comune anche di molti che non ne capiscono a fondo il significato, capiscono ancor meno le difficoltà di applicazione, non di rado li utilizzano come strumento di comunicazione (si dice per "bucare lo schermo" o per attirare masse di follower della rete). In una società nella quale sembra essersi indebolito lo spirito critico in vasti strati della popolazione, non di rado indipendentemente dai livelli di reddito o di istruzione, l'uso dei mantra può creare grande confusione e soprattutto facili illusioni nel potere della volontà. Al riguardo, si ricordano alcune tipiche affermazioni sempre più ricorrenti: "Per risolvere i rilevanti problemi del SSN basta la volontà politica di risolverli"; "Bisogna smetterla di parlare di crisi del SSN, ma occorre saper trasformare i vincoli in opportunità"; "É possibile contemperare la sostenibilità economica di breve e di lungo periodo recuperando ampi spazi di efficienza"; "La competizione tra pubblico e privato nell'erogazione dei servizi di tutela della salute non genera distorsioni in presenza di privato non profit o di privato che accetta un profitto equo e giusto".

Il secondo aspetto da considerare attiene alla relazione tra teorie, politiche, attuazioni, controllo. Non è detto che teorie corrette si traducano in politiche sui sistemi di tutela della salute adeguati. Spesso per semplificazione, o per semplicismo, qualcuno sostiene che per migliorare il livello di appropriatezza, efficacia, efficienza, economicità non è necessario inventare soluzioni, basta adottare politiche che si sono dimostrate efficaci in altri Paesi. Soluzione che sottovaluta il fatto che specie nel campo della tutela della salute i fattori culturali, sociali, religiosi economici, istituzionali sono assai più rilevanti rispetto ai beni o servizi cosiddetti "di massa" e che quindi ogni politica deve essere costruita come un vestito su misura. Infine, in Paesi nei quali domina una cultura formale, spesso si pensa che l'adozione di politiche o di leggi, ancorché valide, rappresentino di per sé la soluzione poiché vige il principio che "le norme devono essere applicate e rispettate". Tutti sanno che non è così, tutti ripetono che non basta decidere le politiche e fare le leggi per cambiare $i$ comportamenti, ma poi ogni volta che si vuole risolvere un problema si approva una nuova legge o si definisce una nuova politica. Questa è l'ultima declinazione del mantra, continuare a fare ciò che tutti dicono che non si dovrebbe fare. 